\title{
ALIANZAS ENTRE EL CONOCIMIENTO BIOLÓGICO Y EL PENSAMIENTO AMBIENTAL. UNA INVESTIGACIÓN CURRICULAR.
}

\section{ALLIANCES BETWEEN THE BIOLOGICAL KNOWLEDGE AND THE ENVIRONMENTAL THOUGHT: A CURRICULAR INVESTIGATION.}

\author{
Carlos Alberto Chacón Ramírez ${ }^{1}$, Mario de Jesús Villada Suaza ${ }^{1}$, Nhora Nitzanim Santa Jiménez ${ }^{2}$ \\ ${ }^{1}$ Docente programa Licenciatura en Biología y Educación Ambiental, Universidad del Quindío \\ ${ }^{2}$ Egresada Licenciatura en Biología y Educación Ambiental.
}

Fecha de recibido: Febrero 3 de 2010

Fecha de aceptado: Junio 9 de 2010

Correspondencia: Programa Licenciatura en Biología y Educación Ambiental, Universidad del Quindío Av. Bolívar calle 12 norte Armenia. Correo electrónico: carloschacon@uniquindio.edu.co

\section{RESUMEN}

El artículo muestra como la investigación busca generar uniones creadoras entre el pensamiento ambiental y el saber biológico, conectados a la organización curricular en la Licenciatura en Biología y Educación Ambiental de la Universidad del Quindío-Colombia. Presenta diagramas y espectros teóricos, los cuales contienen concepciones diversas de orden estético, poético, filosófico y epistémico, que se funden al logos biológico. Todo esto señala, que es posible incorporar pensamiento ambiental en la práctica-praxis educativa, para fundar un nuevo maestro y lograr un currículo reformulado.

PALABRAS CLAVE: pensamiento ambiental, curriculum, saber biológico, estética, poética, erótica, epistemología.

\begin{abstract}
This composition shows some of the achievements reached through an investigative process, which purpose was to generate creative avenues between environmental thought and biological knowledge. That is related to the curriculum organization in the "Licenciatura en Biología y Educación Ambiental" program at University of Quindio-Colombia. Besides, there are supporting diagrams and theoretical spectra, which comprise a diversity of conceptions as stetics, poetics and epistemological so bound to biological logos. It all points out to possibilities to incorporate environmental thoughts in the educative praxispractice. The aim is to create a new teacher and a redesigned curriculum.
\end{abstract}

Key words: Environmental thought, curriculum, biological knowledge, stetic, poetic, epistemological.

\section{INTRODUCCIÓN.}

El proceso investigativo de carácter curricular, muestra como el pensamiento ambiental, se incorpora a los contenidos programáticos de la malla curricular en el Programa de Licenciatura en Biología y Educación Ambiental en la Universidad del Quindío; y, como ejemplo, para el presente documento, se hace referencia a dos microcurriculums, también llamados materias, asignaturas o espacios académicos de carácter biológico: biología molecular y limnología. Es así, que la Investigación indica como es posible, que conceptos y definiciones del logos biológico, se enlacen con rasgos del pensamiento ambiental en términos simbólicos, estéticos, epistémicos, poéticos y, otros que lo caracterizan, a través de lecturas reflexivas, diagramas fundantes y alianzas de pensamiento; todo esto, a partir de una actuación colegiada maestro-estudiante en el ejercicio escolar.

\section{PROBLEMATIZACIÓN.}

El programa de formación, tiene como base disciplinar la biología, y como propósito educativo fundamental la educación ambiental; pero esta última, no había sido considerada en una dimensión formativa que colocara en peso equivalente los dos tipos de saberes. Por este motivo, la investigación se propuso ir al encuentro con maneras de generar alianzas entre el pensamiento ambiental con los saberes biológicos propios del campo de formación; por supuesto, en respeto por sus pisos epistemológicos, pero en franca lid de hallar conexiones criticas, ampliadas y complejizantes, que permitieran permear los contenidos disciplinares y proporcionar otras miradas del mundo de la vida, considerados propicios en la formación de un Licenciado en Biología y Educación Ambiental. 


\section{REFLEXIONES.}

\subsection{El maestro y el pensamiento ambiental.}

La Investigación busca alentar al maestro a cobrar visiones de mundo, desde la biología y el pensamiento ambiental. Pensamiento ambiental, que tiene como mesetas, comprender las inmensas y complejas relaciones que se suscitan en el mundo de la vida, las formas como el ser humano habita la tierra y la comprensión misma del devenir de la vida. Gesta investigativa que promueve a que el maestro y el estudiante, pasen de la alquimia y la mezcla, al relato de la vida que emerge y se incorpora a la existencia, para el cierre del bostezo academicista que los sujeta. En otras palabras, seres humanos preparados a construir comprensión del mundo de la vida no cosificada, desde donde es posible, un ejercicio fluctuante de permanente interrogación sobre la naturaleza, la sociedad, la cultura, la vida $y$, con ellas, sus movilidades, sus conexiones y sus tragedias.

En este sentido, es una investigación que reconoce al maestro como ser humano capaz de colmarse de condiciones onto-eco-lógicas, que le posibiliten lecturas de la complejidad creciente del mundo de la vida $y$, así, refundarlas en su labor escolar. Son pues, maestro y estudiante, invitados a rotar de la episteme dura del signo y la palabra científica, a una episteme recreada por la figura y el símbolo. Dicho de otra manera, seres humanos no abstraídos a reconocer el mundo de los objetos y, a su vez, sin miedo al mito, al símbolo, a la afectividad, a la imaginación, y a desplegar la cualidad de la fascinación, la sensibilidad, la pasión, la curiosidad.

\subsection{Pensamiento ambiental en conquista de la gesta curricular.}

El pensamiento ambiental, proporciona rutas sinuosas para trascender la mera construcción asignaturista, a través de diversas nociones, conceptos, saberes; contándose entre ellos: la ecología profunda, la teoría general de sistemas, el pensamiento complejo, la hipótesis Gaia, la teoría del caos, el pensamiento ecosófico (Guattari 1998:30) 1", y muchos más, los cuales dinamizan la tarea de construir pensamiento ambiental. Nociones metafóricas como la del "rizoma"² (Deleuze, G. y, Guattari, F. 1994:25), del cual se deriva el pensamiento rizomático, como posibilidad de pensar en relación, en red, en coligación, en enlace, a manera de trenza entre naturaleza y cultura, physis y logos. También, pensar desde los matices estéticos, puesto que, como expresa (Noguera, A. 2000:20), "ni lo estético ni lo ambiental niegan la necesidad de una racionalidad ampliada; lo estético insiste en la existencia de otras dimensiones y formas de ser, con las cuales debe entrar en dialogo la racionalidad, no para abarcarlas sino para entenderlas como ellas son." ${ }^{\prime \prime}$. Por estas razones, la organización curricular, marcada por el conocimiento científico, se aboca a zurcir red en coalición estética, porque tanto la estética como el concepto, se apoyan en las metamorfosis, que caracterizan lo vivo. Aquí, los seguros otorgados por el edificio incólume, que guarda lo inamovible de la ciencia, abrirá sus lados para recomponerse y dejar habitar al humano reformado, en paisajes de migración causada por las movilidades del mundo de la vida. Saber biológico y pensamiento ambiental, desde esferas complejizantes, que entienden, que el dejo de la sola respiración fisiológica, no es suficiente para vivir, vivenciar, pervivir en el mundo y, que requiere además, sensibilidad estética, erótica, poética y simbólica en forma de junturas creadoras; como dice (Derrida 1978:81-82) "brisure/juntura. (...) Articulación por medio de una bisagra de dos partes de una obra de carpintería. (...) La juntura de un postigo, (...) origen de la experiencia del espacio y del tiempo ${ }^{\prime 4}$.

\section{METODOLOGÍA.}

Para efecto de ilustrar lo sucedido en la investigación, se presenta lo logrado en dos espacios académicos: biología molecular y limnología. Los mismos, tuvieron similar tratamiento que los demás microcurriculums de la malla curricular. A continuación los pasos que constituyeron la organización de la indagación y los hallazgos:

4.1 Estudio del microcurriculum: (nombre dado a las comúnmente llamadas materias, espacios académicos o asignaturas), en los cuales se aborda un tema biológico posible de ser enlazado con el pensamiento ambiental.

4.2 Motivación: encuentros personales con los maestros para la explicación del proceso investigativo, orientación y diálogo sobre los diferentes campos del pensamiento ambiental posibles de aliarse al conocimiento biológico, los cuales se muestran en el siguiente diagrama organizador (diagrama 1).

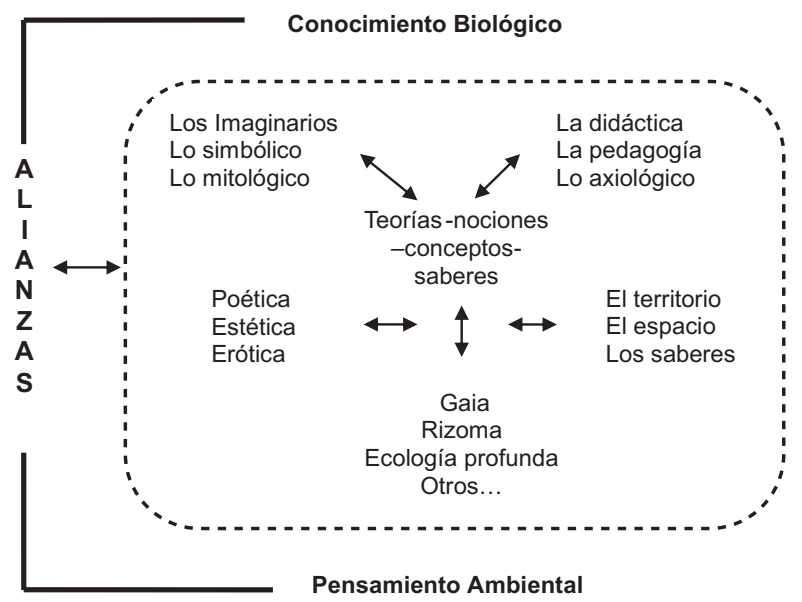

Diagrama 1. Diagrama organizador, que muestra condiciones del pensamiento ambiental como teorías, nociones, conceptos, mitos, símbolos, imaginarios, y otros, que pueden aliarse al conocimiento biológico, a partir de lectura de textos, diálogo, práctica, praxis, saberes y vivencias y, que a la vez, se incorporan al microcurriculum. 
4.1 Escrito convocado: reflexión e interpretación a partir de libros, capítulos de libro o artículos, los cuales se sugieren para el ejercicio académico.

4.2 Diagrama fundante: gráfica originada por la Interpretación de los documentos, que muestra a manera de rutas, las interconexiones entre el conocimiento biológico y condiciones del pensamiento ambiental.

4.3 Alianzas: reflexión causada, en términos de los lazos posibles entre el conocimiento biológico y el pensamiento ambiental.

\section{ALIANZAS ENTRE EL CONOCIMIENTO BIOLÓGICO Y EL PENSAMIENTO AMBIENTAL. Casos: biología molecular y limnología.}

\subsection{Desde la biología molecular; la inmunología y el cuerpo.}

5.1.1 Estudio del microcurriculum: a manera de definición: la biología molecular, es la rama de la biología que investiga las causas moleculares de los fenómenos biológicos. A partir de su estudio, se localizan los temas que muestran posibilidades de alianza al pensamiento ambiental, en este caso se elige el sistema inmune.

5.1.2 Motivación: la intención es que el estudio de la biología molecular $y$, uno de sus temas fundamentales como es la inmunidad, sean exhortados para re-apreciar el cuerpo y considerarlo como portador de un primer registro del ser. Registro del ser, por el cual, el cuerpo se conecta con el afuera de su corporeidad física. Esto, porque relacionarse con el mundo, admite universos pletóricos de símbolos e imaginarios, los cuales no son de ningún modo abstractos, ni únicamente racionales o utilitarios, sino que se encuentran rubricados por sentimientos y experiencias, que no tienen que ver con la rigidez de la razón instrumental.

5.1.3 Escrito convocado: el cuerpo evocador, una relectura de la inmunidad, en el libro, el fenómeno de la vida, de Francisco Varela, 2002.

Con su lectura, se desprenden diversidad de junturas poiésicas; es decir, creadoras, a manera de despliegues conceptuales y sensibles de los participantes, para ver como el sistema inmunitario se resignifica en el cuerpo. De tal fricción de saberes, surge el diagrama fundante (diagrama 2), como holograma, que muestra rutas posibles y condición de alianza entre el saber biológico y el pensamiento ambiental.

\subsubsection{Diagrama fundante:}

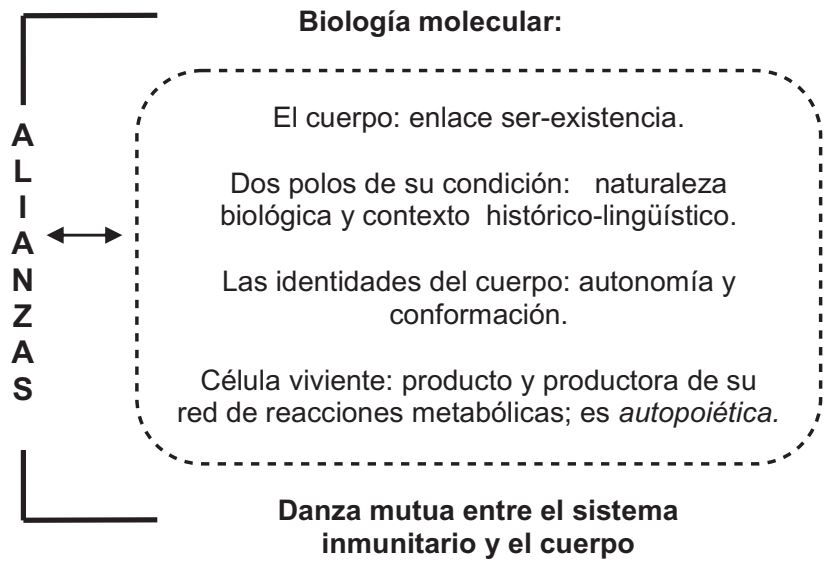

Diagrama 2. Junturas poiésicas/creadoras entre el cuerpo orgánico-inmune con el cuerpo como ser-existencia. Danza mutua entre el sistema inmunitario y el cuerpo, que permite al cuerpo poseer una identidad cambiante y plástica a lo largo de su existencia. Pensamiento que permite una relectura de la inmunidad.

5.1.5 Alianzas : cuerpo que genera conexiones con el mundo de la vida, a partir de sus funciones antígeno-sistema inmune en identidad somática, adherida indeleblemente a las experiencias y actividades más familiares y cotidianas. Una relectura desde el interior del cuerpo, la inmunidad y sus "sistemas autopoiéticos" (Varela, 2002:54).

Cuerpo que despliega autonomía y construye sentido, en deseo de no renunciar a reconocer las redes, en las que soporta su condición, en tanto cultura y sociedad. Se trata, entonces, de disponer en crisis la cognición, para deconstruir la creencia de una existencia corpórea, orgánica sin más. Así pues, es necesario circular por agendas discontinuas, que permitan un método que ame la vida y descubra su fecundidad; es decir, una dimensión estética que nos haga vibrar en común y ser identitarios de este mundo, en franca lid dialógica con el concepto. En otras palabras en una erótica del conocimiento, tal como lo menciona (Maffesoli 1996:168) "recuerda que al lado de la brutalidad del concepto, que cree agotar aquello mismo a lo que se aproxima, vaciando, en nombre de la eternidad, el aspecto frágil de las cosas, puede existir otra aproximación, mucho mas acariciadora, atenta al detalle, a los elementos menores, en una palabra, a lo que esta vivo" ${ }^{\prime 6}$. De esta manera, reconocer el cuerpo, como la morada más íntima, la morada mutante, la morada vital.

\subsection{Desde la limnología; el agua en poética recreada.}

5.2.1 Estudio del microcurriculum: a manera de definición resumida, la limnología es la rama de la ecología que estudia los ecosistemas acuáticos continentales (lagos, lagunas, ríos, charcas, marismas y estuarios). Desde ella, se localizan los temas que muestran posibilidades de alianza al pensamiento ambientaly, para el efecto, se elige el agua. 
5.2.2 Motivación: el agua en sentido de vitalidad terrestre. Agua que trasciende la fórmula molecular, para situarla en espacios pletóricos de inmanencias y remembranzas. El agua que hace posible la trama de la vida, deviene intersubjetividad gozosa, a la manera de atracción lúdica y sensorial y, que a su vez, hace lenguaje el amor a la vida e incorpora ensoñación.

5.2.3 Escrito convocado: libro, el agua y los sueños; obra de Gaston Bachelard, 1978.

Aquí, el triatomismo molecular se traduce en líquido mayor, en líquido omnipresente, un líquido en supremacía sobre esta tierra viva y de todos. En palabras del autor invitado (Bachelard 1978:235) "¿...cómo decir más claro que la intuición soñadora del agua persiste a pesar de las circunstancias adversas?. El agua del cielo, la fina lluvia, la fuente amiga y salutífera dan lecciones más directas que todas las aguas de los mares. (...) La ensoñación natural guarda siempre un privilegio para el agua dulce, para el agua que refresca y quita la sed". Es así, que el texto invita a capturar la quietud, lentitud y profundidad abisal de las aguas; de las aguas primaverales, las aguas claras, las aguas corrientes, en erótica-estética-ensoñación, mostrados en el siguiente diagrama fundante (diagrama3):

\subsubsection{Diagrama fundante.}

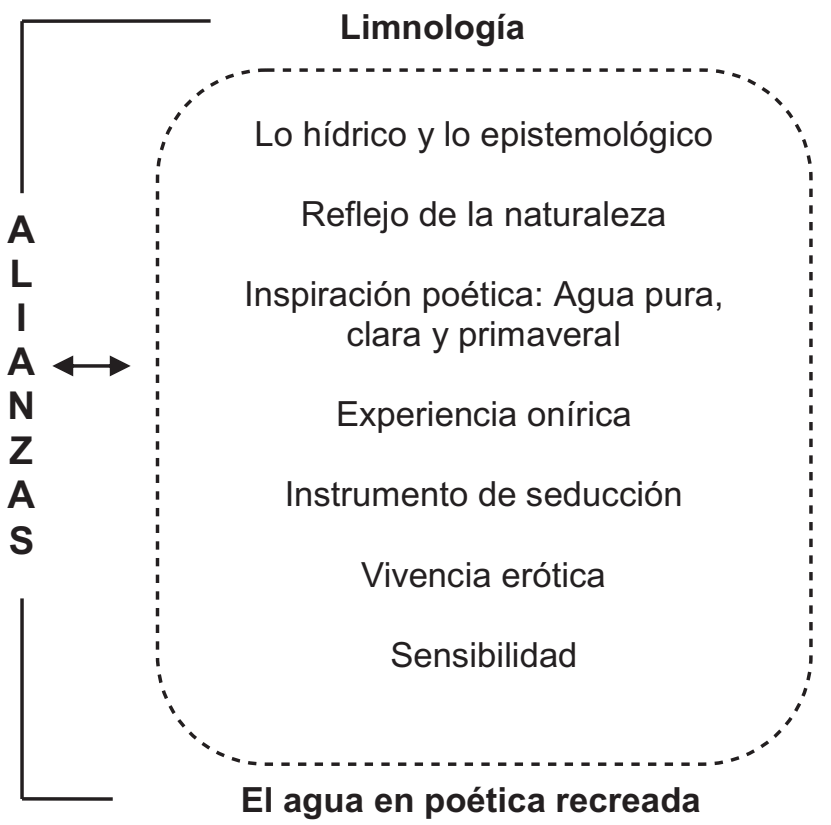

Diagrama 3. Contenidos epistemológicos, estéticos, eróticos, vitales, sensibles que se dilatan y conectan al logos biológico, a manera de enlaces de sentido. El agua expresada como vivencia en poética recreada.
5.2.5 Alianzas: su cientificidad, en tanto recurso natural está presente, los pisos científicos sobre los que se soporta, son ampliamente estudiados, y la ciencia normal se ocupa permanentemente por su disponibilidad, uso, conservación y almacenamiento. Sin embargo, no son suficientes para entenderla en sus dimensiones de conexión con la vida. Es crucial, entonces, trasladarse por ella desde condiciones ético-estéticas-poético-eróticas-oníricas, apreciar las cualidades fenomenológicas cuando luce expuesta en escena natural y considerarla provocadora de lenguajes paralelos entre la sensualidad, la sensibilidad y la imaginación.

Agua en diferentes estados físico-químicos, del cual surgen diversidad de pensamientos, que van desde la referencia exclusivamente molecular, hasta expresiones fenomenológicas como líquido sublime, líquido vital. Correlatos de experiencias oníricas emergidas del contacto con el compuesto natural, que reconocen su necesaria vitalidad, a manera de emocionalidad hídrica; en otras palabras, en una especie de acuosidad seductora.

\section{EMERGENCIASDE LA INVESTIGACIÓN.}

Movilidades de pensamiento y reformulación de los microcurriculums, a partir de la lectura y el ejercicio reflexivo, que incorpora pensamiento y complejidad ambiental aliados al conocimiento biológico.

Los maestros y estudiantes, tienen a través de esta investigación curricular, la oportunidad de acceder a renovadas formas de comprender el ambiente, desde esferas complejizantes y panoramas de contexto.

\section{EPÍLOGO.}

Maestro dispuesto a superar miradas de mundo estáticas y enciclopédicas en sus actuaciones escolares. Llamado, a que el saber biológico, físico, químico, matemático; en fin, el conocimiento inveteradamente adscrito a la racionalidad tecno-científica, sea llamado a una pragmática reflexiva en las múltiples relaciones del mundo de la vida. Invitación a que ocurran traslados de pensamiento, desde los conocimientos propios de la formación de este profesional de la educación u, otros de diversa índole, hacia rasgos del pensamiento ambiental, lo que puede movilizar no sólo su pensamiento, sino el diseño de sus prácticas educativas. 
BIBLIOGRAFÍA.

Bachelard, G. (1978). La poética del espacio. México: Fondo de Cultura Económica.

(1978). El Agua y los Sueños. México: Fondo de Cultura Económica.

Capra, F. (2000). La trama de la vida. Una nueva perspectiva de los sistemas vivos. Barcelona: Anagrama.

Deleuze G. y Guattari, F. (1994). Mil mesetas. Capitalismo y esquizofrenia. Valencia: Pre-textos.

Guattari, F. (1998). Las tres ecologías. Valencia: Pre-textos.

Maffesoli, M. (1996). Elogio de la razón sensible. Una visión intuitiva del mundo contemporáneo. Barcelona: Paidós.

Morín, E. (1999). La cabeza bien puesta. Buenos Aires: Ediciones Nueva Visión SAIC.

Noguera, A.P. (2000). Educación estética y complejidad ambiental. Bogotá: Universidad Nacional de Colombia.

Varela, F. (2002). El fenómeno de la vida. Santiago de Chile: Dolmen Ediciones S.A. 